\title{
Avaliação das Condições Meteorológicas Simuladas pelo Modelo WRF na Região Metropolitana do Rio de Janeiro em Dias Com Altas Concentrações de Poluentes
}

\author{
Wilson Willian da Silveira $^{1}$ (D) , Vanessa Silveira Barreto Carvalho ${ }^{1}$ \\ ${ }^{1}$ Instituto de Recursos Naturais, Universidade Federal de Itajubá, Itajubá, MG, Brasil.
}

Recebido em: 9 de Junho de 2019 - Aceito em: 1 de Setembro de 2019

\begin{abstract}
Resumo
Frequentemente, condições meteorológicas desfavoráveis à dispersão de poluentes em conjunto com altas taxas de emissões de poluentes atmosféricos e uma topografia complexa favorecem a ocorrência de altas concentrações de poluentes na Região Metropolitana do Rio de Janeiro (RMRJ). Nesse contexto, esse estudo verificou a influência de condições meteorológicas em dois períodos onde se verificou a ocorrência de altas concentrações de $\mathrm{O}_{3}$ e $\mathrm{PM}_{10}$ em dias consecutivos na RMRJ. O estudo utilizou o modelo numérico de mesoescala Weather Research and Forecanting Model (WRF) para simulação das condições e variáveis meteorológicas favoráveis para manutenção desses episódios, verificando a influência do Anticiclone Subtropical do Atlântico Sul (ASAS), de altos valores de temperatura do ar, baixos valores de velocidade do vento e ausência de nebulosidade, em concordância com os dados observados. Através da análise visual foi possível verificar que o modelo foi eficaz na representação do ASAS, precipitação e nebulosidade. O modelo apresentou correlação linear de Pearson positiva entre a temperatura simulada e observada acima de 0,71 nos 2 períodos em todas as 8 estações meteorológicas e correlação linear de Pearson positiva entre a umidade relativa do ar simulada e observada acima de 0,72 . O cálculo do erro médio (ME) indicou que, em geral, o modelo subestimou a temperatura do ar e superestimou a umidade relativa do ar. A aplicação dos métodos estatísticos aos dados observados e simulados pelo modelo revelou que o modelo possui habilidade (skill) em representar as variáveis meteorológicas para a maioria das estações.
\end{abstract}

Palavras-chave: ozônio, material particulado, região metropolitana, WRF.

\section{Evaluation of the Meteorological Conditions Simulated by the WRF Model in the Metropolitan Area of Rio de Janeiro on Days with High Concentrations of Pollutants}

\begin{abstract}
Often, meteorological conditions that are unfavorable to the dispersion of pollutants together with high atmospheric pollutant emissions and a complex topography favor the occurrence of high concentrations of pollutants in the Metropolitan Area of Rio de Janeiro (MARJ). In this context, this study verified the influence of meteorological conditions in two periods where the occurrence of high concentrations of $\mathrm{O}_{3}$ and $\mathrm{PM}_{10}$ was observed on consecutive days in MARJ. The study used the numerical model Weather Research and Forecanting Model (WRF) to simulate the conditions and favorable meteorological variables to maintain these episodes, verifying the influence of the South Atlantic Subtropical Anticyclone (SASA), high air temperature, low values of wind speed and absence of cloudiness, in agreement with the data observed. Through the visual analysis, it was possible to verify that the model was effective in the SASA representation, precipitation and cloudiness. The model presented positive of the Pearson linear correlation between simulated and observed temperature above 0.71 in the 2 periods in all 8 meteorological stations and a positive Pearson linear correlation between simulated and observed air humidity above 0.72 . The calculation of the mean error (ME) indicated that, in general, the model underestimated the air temperature and overestimated the relative humidity of the air. The application of the statistical methods to the data observed and simulated by the model revealed that the model has the skill to represent the meteorological variables for most stations.
\end{abstract}

Keywords: ozone, particulate material, metropolitan area, WRF.

Autor de correspondência: Wilson Willian da Silveira, wilson.silveira.w@gmail.com. 


\section{Introdução}

As megacidades, regiões de aglomerados populacionais com mais de 10 milhões de habitantes, representam grandes fontes de poluição atmosférica (Lawrence et al., 2007). A América do Sul possui 7 megacidades, dentre as quais encontra-se a Região Metropolitana do Rio de Janeiro (RMRJ) (Gallardo, 2012). A RMRJ abrange 19 municípios, possui uma população de 12,5 milhões de habitantes (IBGE, 2016) e apresenta a segunda maior frota veicular e concentração industrial do país. As fontes veiculares são responsáveis por $77 \%$ das emissões de poluentes atmosféricos na região enquanto estima-se que as fontes de emissão industriais correspondem a $23 \%$ do total (INEA, 2015).

No que se refere a qualidade do ar, é importante destacar que o Brasil possui uma rede de monitoramento ainda limitada; a RMRJ, por exemplo, possui 35 estações de monitoramento das quais 27 registram concentrações de material particulado com diâmetro inferior a $10 \mu \mathrm{m}$ $\left(\mathrm{PM}_{10}\right)$ e 18 de ozônio $\left(\mathrm{O}_{3}\right)$ (INEA, 2015). Logo, dada a extensão da região, a topografia complexa, a presença de grandes corpos d'água e localização costeira, além da diversidade das fontes emissoras, verifica-se um número insuficiente de estações de qualidade do ar.

As estações existentes, frequentemente, registram índices de concentrações de poluentes acima dos Padrões Nacionais de Qualidade do Ar (PNQA) estabelecidos pela resolução CONAMA 03/90 como seguros para a população, principalmente no que se refere as concentrações de $\mathrm{PM}_{10}$ e de $\mathrm{O}_{3}$ (INEA, 2014; INEA, 2015). Porém, é importante destacar que a Resolução CONAMA 03/90 foi revogada em 19 de novembro de 2018 e, em seu lugar, foi implementada a Resolução CONAMA 491/18, que tem como meta atingir, gradualmente, os limiares de referência recomendados pela Organização Mundial de Saúde (OMS, 2006) como PNQA de forma a garantir a saúde e o bemestar da população. A meta final é alcançar médias móveis de 8 horas de $100 \mu \mathrm{gm}^{-3}$ de ozônio, e médias de 24 horas, diárias, de $50 \mu \mathrm{g} \cdot \mathrm{m}^{-3}$ de $\mathrm{PM}_{10}$, considerados seguros para saúde da população. Ainda não existem estudos sobre o impacto da adoção dos novos padrões na RMRJ. Contudo, Santos et al. (2016) e Silveira (2019) identificaram períodos com valores acima dos valores de referência estabelecidos pela OMS para $\mathrm{O}_{3}$ e $\mathrm{PM}_{10}$. Vale ressaltar que diversos estudos associaram o agravamento de doenças cardiovasculares, respiratórias e neurológicas, principalmente em crianças e idosos, aos níveis de poluição a que uma pessoa é exposta (Fajersztajn et al., 2017; Takano et al., 2019).

Também é importante enfatizar que as condições meteorológicas exercem grande influência na qualidade do ar sendo essas diretamente responsáveis pelo transporte e dispersão de poluentes primários, poluentes que são emitidos diretamente na atmosfera, podendo também influen- ciar na formação e dispersão de poluentes secundários na atmosfera, como é o caso do $\mathrm{O}_{3}$, principalmente devido as variáveis meteorológicas como temperatura, velocidade dos ventos, radiação, nebulosidade, umidade relativa do ar, entre outras (Carvalho et al., 2012; Santos et al., 2016). Nesse contexto, a utilização de modelos numéricos de mesoescala pode fornecer subsídios para a avaliação dos impactos de diferentes condições meteorológicas na qualidade do ar. O objetivo principal deste estudo é avaliar as condições meteorológicas durante dias quando episódios de concentração de $\mathrm{PM}_{10}$ e $\mathrm{O}_{3}$ foram registrados na RMRJ, a partir da utilização do modelo de mesoescala WRF (Weather Research and Forecanting Model).

\section{Material e Métodos}

\subsection{Dados utilizados}

Foram utilizados dados de concentração dos poluentes $\mathrm{PM}_{10}$ e $\mathrm{O}_{3}$, e também das variáveis meteorológicas de direção e velocidade do vento, temperatura do ar, umidade relativa do ar e precipitação monitorados pela Secretaria de Meio Ambiente do Município do Rio de Janeiro (SMAC). Neste estudo foram consideradas 8 (oito) estações de monitoramento da qualidade do ar cuja distribuição espacial está apresentada na Fig. 1.

Para a seleção dos períodos a serem simulados com o modelo WRF foram analisados dados de concentração de $\mathrm{O}_{3}$ e $\mathrm{PM}_{10}$ com frequência amostral de 10 em 10 minutos, para o período de 2012 a 2015. Para comparação com os valores de referência estabelecidos pela OMS (2006), valores equivalentes a Meta Final da Resolução CONAMA 491/18, foram calculadas médias móveis de $8 \mathrm{~h}$ para o $\mathrm{O}_{3}\left(100 \mu \mathrm{g} \cdot \mathrm{m}^{-3}\right)$, a partir das quais foi extraído o maior valor do dia, e médias de $24 \mathrm{~h}$ para o $\mathrm{PM}_{10}\left(50 \mu \mathrm{g} \cdot \mathrm{m}^{-3}\right)$. A partir destas informações foram selecionados 2 períodos de estudo nos quais foram registrados em dias consecutivos médias máximas superiores aos valores de referência considerados neste estudo.

O primeiro período (17 a 22 de novembro de 2013) apresentou concentrações máximas das médias móvel de $8 \mathrm{~h}$ de $\mathrm{O}_{3}$ acima de $100 \mu \mathrm{g} \cdot \mathrm{m}^{-3}$, em pelo três das estações de monitoramento durante os dias de estudo e concentrações médias diárias de $\mathrm{PM}_{10}$ acima $50 \mu \mathrm{g} . \mathrm{m}^{-3}$, em pelo menos uma das estações de monitoramento. $\mathrm{O}$ segundo período de estudo (23 a 27 de setembro de 2015) apresentou concentrações médias móvel de $8 \mathrm{~h}$ de $\mathrm{O}_{3}$ acima dos $100 \mu \mathrm{g} . \mathrm{m}^{-3}$, em pelo menos três das estações em análise durante o decorrer dos quatros dias analisados e concentrações médias diária de $\mathrm{PM}_{10}$ acima $50 \mu \mathrm{g} \cdot \mathrm{m}^{-3}$, em pelo menos três das estações de monitoramento. As maiores concentrações de $\mathrm{O}_{3}$ e $\mathrm{PM}_{10}$ registradas na RMRJ para os dois períodos de estudo podem ser observadas nas Tabelas 1 e 2, respectivamente. Durante ambos os períodos é possível verificar que a concentração de $\mathrm{O}_{3}$ ultra- 




Figura 1 - Distribuição espacial das estações de monitoramento consideradas na RMRJ. Onde: (1) Copacabana, (2) São Cristóvão, (3) Centro, (4) Tijuca, (5) Irajá, (6) Bangu, (7) Campo Grande e (8) Pedra Guaratiba (Fonte: Google Earth).

passa também os limites estabelecidos pela resolução CONAMA 491/18 atualmente em vigor, com valores superiores de $140 \mu \mathrm{g} \cdot \mathrm{m}^{-3}$ considerando a média móvel de $8 \mathrm{~h}$, durante alguns dias. Durante o segundo período também é possível verificar a ultrapassagem dos limites em vigor estabelecidos pela resolução CONAMA 491/18 para $\mathrm{PM}_{10}$, de $120 \mu \mathrm{g} . \mathrm{m}^{-3}$, durante dois dias.

A análise e verificação das condições de grande escala predominantes foi feita através de cartas sinóticas da Marinha do Brasil disponibilizadas pela Diretoria de Hidrografia e Navegação da Marinha do Brasil, disponível em seu sítio na internet. Foram utilizados também dados de cobertura de nuvens obtidos por meio do METAR de estações dos 5 aeroportos da RMRJ, para análise dos períodos e comparação como os dados simulados, disponíveis no site da Rede de Meteorologia do Comando da Aeronáutica - REDEMET.

\subsection{Configurações e validação do modelo}

Para simulação das condições meteorológicas durante o período de estudo foi utilizado o modelo meteoro-

Tabela 1 - Máximas Concentrações de $\mathrm{O}_{3}$ e $\mathrm{PM}_{10}$, em $\mu \mathrm{g} . \mathrm{m}^{-3}$, registradas na RMRJ durante o período de 17/11/2013 a 22/11/2013.

\begin{tabular}{lllllll}
\hline & \multicolumn{6}{c}{ Data } \\
\cline { 2 - 7 } $\begin{array}{l}\text { Máxima } \\
\text { concentração }\end{array}$ & $17 / 11$ & $18 / 11$ & $19 / 11$ & $20 / 11$ & $21 / 11$ & $22 / 11$ \\
\hline $\mathrm{O}_{3}$ & 102,50 & 105,81 & 142,38 & 134,29 & 166,30 & 118,70 \\
$\mathrm{PM}_{10}$ & 53,94 & 38,87 & 63,07 & 70,90 & 73,63 & 41,93 \\
\hline
\end{tabular}

lógico de mesoescala WRF. O WRF resolve equações físicas, dinâmicas e computacionais e pode ser aplicado para previsão do tempo, estudos de assimilação de dados e modelos geofísicos acoplado (Skamarock et al., 2008). Os dados do Global Forecast System (GFS) com $0,5^{\circ}$ de resolução espacial, utilizados para a inicialização e geração das condições de fronteira das simulações, foram obtidos através do National Climatic Data Center do National Oceanic and Atmospheric Administration.

As simulações com o WRF foram feitas com duas grades com resolução de $16 \mathrm{~km} \mathrm{e} 4 \mathrm{~km}$, respectivamente, e 32 níveis verticais, ambas centradas em $22,8^{\circ} \mathrm{S}$ e $43,25^{\circ} \mathrm{W}$ na RMRJ. As configurações do modelo WRF e parâmetros físicos estão disposto na Tabela 3. Todas as simulações foram disparadas com $24 \mathrm{~h}$ de antecedência ao período de interesse para a eliminação do efeito de spin-up do modelo.

A verificação dos resultados das simulações foi realizada através da comparação destes com os dados observados de temperatura e umidade relativa do ar. Para isso, foram calculados: o desvio padrão $(\sigma)$ das séries observa-

Tabela 2 - Máximas concentrações de $\mathrm{O}_{3}$ e $\mathrm{PM}_{10}$, em $\mu \mathrm{g} . \mathrm{m}^{-3}$, registradas na RMRJ durante o período de 23/09/2015 a 27/09/2015.

\begin{tabular}{lccccc}
\hline & \multicolumn{5}{c}{ Data } \\
\cline { 2 - 6 } Máxima concentração & $23 / 9$ & $24 / 9$ & $25 / 9$ & $26 / 9$ & $27 / 9$ \\
\hline $\mathrm{O}_{3}$ & 157,15 & 142,24 & 134,99 & 116,80 & 146,00 \\
$\mathrm{PM}_{10}$ & 83,19 & 131,80 & 126,63 & 71,77 & 73,68 \\
\hline
\end{tabular}


Tabela 3 - Configuração do modelo e parametrizações físicas.

\begin{tabular}{lcc}
\hline \multicolumn{1}{c}{ Parametrização } & Grade 1 & Grade 2 \\
\hline Pontos na direção X & 61 & 53 \\
Pontos na direção Y & 30 & 25 \\
Níveis verticais & 33 & 33 \\
Resolução horizontal & $16 \mathrm{~km}$ & $4 \mathrm{~km}$ \\
Latitude central & \multicolumn{3}{c}{$-22.80^{\circ}$} \\
Longitude central & \multicolumn{3}{c}{-43.25} \\
Microfísica & \multicolumn{3}{c}{ Lin } \\
Cúmulos & \multicolumn{3}{c}{ Kain-Fritsch } \\
Camada limite planetária & \multicolumn{3}{c}{ YSU } \\
Camadas do solo & \multicolumn{3}{c}{ Monin-Obukhov } \\
Radiação de onda curta & Dudhia \\
Radiação de onda longa & \multicolumn{3}{c}{ RRTM } \\
\hline
\end{tabular}

das e simuladas, o coeficiente de correlação de Pearson (r), o erro médio $(\mathrm{ME})$ e a raiz do erro médio quadrático (RMSE) (Wilks, 1995). O índice de concordância (d) foi calculado de acordo com Elbir (2003). Também foi calculada a raiz do erro médio quadrático com remoção do desvio médio $\left(\mathrm{RMSE}_{\mathrm{rdm}}\right)$ proposto por Pielke (2002), de acordo com a Eq. (1), para avaliar a habilidade (skill) da simulação. Segundo Pielke (2002), a habilidade do modelo é avaliada uma vez que sejam obedecidas as seguintes condições: (1) $\sigma_{\text {sim }} \approx \sigma_{\text {obs }}$; (2) RMSE $<\sigma_{\text {obs }}$; (3) $\mathrm{RMSE}_{\mathrm{rdm}}<\sigma_{\text {obs. }}$

$$
\operatorname{RSME}_{r d m}=\left\{\frac{1}{N} \sum_{i=1}^{N}\left[\left(y_{i}-\underline{y}\right)-\left(o_{i}-\underline{o}\right)\right]^{2}\right\}^{1 / 2}
$$

Para análise da relação meteorologia e qualidade do ar também foram geradas figuras a partir dos dados simulados pelo WRF, a cada uma hora, através do GRADS.

\section{Resultados e Discussão}

A análise das cartas sinóticas revelou a predominância do Anticiclone Subtropical do Atlântico Sul (ASAS), deslocado para oeste de sua posição, durante os períodos selecionados quando altas concentrações de $\mathrm{PM}_{10}$ e $\mathrm{O}_{3}$ foram registradas. Nos dois períodos analisados, a temperatura máxima nas estações consideradas apresentou valores acima de $30^{\circ} \mathrm{C}$; a velocidade média do vento durante a madrugada e de manhã ficou abaixo de $1,97 \mathrm{~m} / \mathrm{s}$, caracterizando a predominância de ventos fracos na região. Também foi possível observar a predominância de condições de céu-claro na maioria dos dias. Tais resultados corroboram os resultados encontrados por Carvalho (2010) e Santos et al. (2016) onde foram analisadas as condições meteorológicas predominantes durantes dias com altas concentrações de $\mathrm{O}_{3}$ e $\mathrm{PM}_{10}$, respectivamente. Vale destacar que sob a influência do ASAS, em geral, verifica-se a ocorrência de ventos fracos, que dificultam a dispersão de poluentes como os precursores de $\mathrm{O}_{3}$ e $\mathrm{PM}_{10}$. A baixa nebulosidade, também associada a atuação do ASAS, influencia a incidência de radiação solar que é fundamental para formação do $\mathrm{O}_{3}$. A temperatura, por sua vez, responde diretamente a incidência de radiação, logo, pode-se associar essa variável meteorológica a formação de $\mathrm{O}_{3}$.

A comparação visual entre as cartas sinóticas e o campo de pressão simulados pelo modelo WRF, através da grade de $16 \mathrm{~km}$, permitiu verificar que modelo simulou bem a configuração do ASAS durante os dois períodos. Assim como o campo de nebulosidade, através dos resultados para a grade de $4 \mathrm{~km}$, revelando a predominância de céu-claro na maioria dos dias, em concordância com as observações. Durante o primeiro período, foi verificada a ocorrência de precipitação moderada (observação e modelo), durante o período da tarde do dia 17 e durante a noite no dia 22. No segundo período foi verificada precipitação na região apenas durante a noite do dia 27 . Vale destacar que a precipitação atua na remoção dos poluentes da atmosfera; contudo, a ocorrência de precipitação em momentos isolados do período não revelou impacto significativo para a melhoria das condições da qualidade do ar na região.

Os resultados obtidos a partir da aplicação dos índices estatísticos para verificação de como o modelo simulou a temperatura são apresentados de forma simplificada na Tabela 4, para os dois períodos de estudo. A correlação linear de Pearson $(\mathrm{R})$ apresentou valores relativamente altos, acima de 0,71 , nas estações de meteorológicas, em ambos os períodos. A estação de Irajá apresentou o maior valor de correlação durante o primeiro período $(0,90)$; já durante o segundo período a estação do Centro foi a que apresentou o maior valor de correlação $(0,84)$. A análise do erro médio revela que o modelo, em média, subestimou os valores de temperatura do ar em todas as estações durante o primeiro período; para o segundo período o modelo superestimou os valores em duas estações, Centro e Tijuca, e subestimou nas demais. Os valores do índice de concordância foram elevados nas estações meteorológicas (acima de 0,74 ), para os dois períodos.

Para o período de 17 a 22 de novembro de 2013, o primeiro critério para obter o skill da simulação, não foi atendido, utilizando uma diferença percentual de até $20 \%$ para avaliação, nas estações da Tijuca e Pedra de Guaratiba, o segundo critério, não foi atendido nas estações de São Cristóvão e Pedra de Guaratiba, e o terceiro, foi atendido em todas as estações. Dessa forma, a habilidade do modelo em prever a temperatura do ar para estações de Copacabana, Irajá, Bangu e Campo Grande foi considerada boa durante esse período de estudo. Para o período de 23 a 27 de setembro de 2015 o primeiro critério para obter o skill da simulação, não foi atendido, considerando como limite uma diferença percentual de até $20 \%$ para avaliação, na estação de Pedra de Guaratiba; o segundo critério, 
Tabela 4 - Análise Estatística da temperatura do ar simulada com a observada para os períodos $\left(1^{\circ}\right)$ de $17 / 11 / 2013$ a $22 / 11 / 2013$ e $\left(2^{\circ}\right) 23 / 09 / 2015$ a $27 /$ $09 / 2015$.

\begin{tabular}{|c|c|c|c|c|c|c|c|c|c|c|c|c|}
\hline \multirow{2}{*}{$\begin{array}{l}\text { Período } \\
\text { Estação }\end{array}$} & \multicolumn{6}{|c|}{$1^{\circ}$} & \multicolumn{6}{|c|}{$2^{\circ}$} \\
\hline & $\mathrm{R}$ & $\mathrm{ME}$ & d & $\mathrm{rmd}$ & $\sigma_{\text {obs }}$ & $\sigma_{\text {sim }}$ & $\mathrm{R}$ & $\mathrm{ME}$ & d & rmd & $\sigma_{\mathrm{obs}}$ & $\sigma_{\text {sim }}$ \\
\hline Centro & - & - & & - & - & & 0,84 & 0,58 & 0,91 & 2,68 & 4,94 & 4,44 \\
\hline Copacabana & 0,79 & $-0,45$ & 0,86 & 2,27 & 3,68 & 2,81 & 0,80 & $-1,23$ & 0,86 & 2,67 & 4,34 & 3,96 \\
\hline São Cristóvão & 0,91 & $-3,77$ & 0,78 & 2,50 & 5,35 & 3,64 & 0,83 & $-0,46$ & 0,91 & 2,66 & 4,46 & 4,73 \\
\hline Tijuca & 0,88 & $-0,73$ & 0,85 & 3,56 & 6,13 & 3,36 & 0,71 & 0,29 & 0,82 & 3,98 & 5,64 & 4,52 \\
\hline Irajá & 0,90 & $-0,92$ & 0,93 & 2,17 & 4,97 & 3,90 & 0,62 & $-1,30$ & 0,74 & 5,05 & 6,30 & 5,04 \\
\hline Bangu & 0,88 & $-1,60$ & 0,88 & 2,40 & 4,92 & 3,67 & 0,72 & $-0,45$ & 0,83 & 3,91 & 5,57 & 4,76 \\
\hline Campo Grande & 0,87 & $-1,40$ & 0,90 & 2,41 & 4,92 & 3,96 & - & - & - & - & - & - \\
\hline Pedra de Guaratiba & 0,71 & $-2,87$ & 0,76 & 3,69 & 4,46 & 1,24 & 0,71 & $-5,17$ & 0,75 & 3,50 & 4,47 & 1,67 \\
\hline
\end{tabular}

não foi atendido também para estação de Pedra de Guaratiba, enquanto o terceiro, foi atendido em todas as estações. Logo, o modelo possui habilidade em simular a temperatura do ar nas estações do Centro, Copacabana, São Cristóvão, Tijuca, Irajá, Bangu e Campo Grande. É possível verificar que ambos os períodos a temperatura não foi bem simulada pelo modelo na estação de Pedra de Guaratiba. A dificuldade em simular as condições locais pode estar relacionado à fatos como a proximidade ao oceano e outros aspectos relativos ao uso do solo não bem representados pelo modelo, principalmente, considerando uma resolução espacial de $4 \mathrm{~km}$.

Para a umidade relativa do ar, durante os dois períodos, o resultado da aplicação dos índices estatísticos é apresentado na Tabela 5. A correlação linear de Pearson (R) apresentou valores, acima de 0,75 , durante o primeiro período, e valores acima de 0,51 , durante o segundo período. As estações da Tijuca e Bangu apresentaram o maior valor de correlação durante o primeiro período $(0,89)$; já durante o segundo período a estação do Centro foi a que apresentou o maior valor de correlação $(0,71)$. A análise do erro médio revela que o modelo subestimou os valores de umidade nas estações de São Cristóvão, Tijuca e Bangu, e superestimou nas demais, durante o primeiro período; para o segundo período, o modelo superestimou os valores em duas estações, Pedra de Guaratiba e Tijuca, e subestimou nas demais. Os valores do índice de concordância nas estações meteorológicas ficaram acima de 0,72, para os dois períodos.

Para o período de 17 a 22 de novembro de 2013, o primeiro critério para obter o skill da simulação, não foi atendido, utilizando uma diferença percentual de até $20 \%$ para avaliação, nas estações de São Cristóvão, Bangu e Pedra de Guaratiba, o segundo critério e o terceiro, foram atendidos em todas as estações. Logo, apenas as estações do Centro, Copacabana, Tijuca e Irajá obedeceram a todos os critérios que caracterizam a habilidade do modelo em representar essa variável durante o período de estudo. Para o período de 23 a 27 de setembro de 2015, o primeiro critério para obter o skill da simulação, não foi atendido, utilizando uma diferença percentual de até $20 \%$ para avaliação, na estação de Pedra de Guaratiba; o segundo critério, não foi atendido para estações de Copacabana, São Cristóvão, Bangu e Pedra de Guaratiba, e o terceiro, não foi atendido para estação de Bangu. Dessa forma, a habilidade do modelo em simular a UR foi verificada nas estações do Centro, Tijuca e Irajá durante o período de estudo.

Outro ponto importante é o papel da brisa marítima no processo de dispersão de poluentes na RMRJ (Car-

Tabela 5 - Análise Estatística da umidade relativa do ar simulada com a observada para os períodos $\left(1^{\circ}\right)$ de 17/11/2013 a 22/11/2013 e $\left(2^{\circ}\right) 23 / 09 / 2015$ a 27/09/2015.

\begin{tabular}{|c|c|c|c|c|c|c|c|c|c|c|c|c|}
\hline \multirow{2}{*}{$\begin{array}{l}\text { Período } \\
\text { Estação }\end{array}$} & \multicolumn{6}{|c|}{$1^{\circ}$} & \multicolumn{6}{|c|}{$2^{\circ}$} \\
\hline & $\mathrm{R}$ & $\mathrm{ME}$ & d & rmd & $\sigma_{\mathrm{obs}}$ & $\sigma_{\mathrm{sim}}$ & $\mathrm{R}$ & $\mathrm{ME}$ & $\mathrm{d}$ & rmd & $\sigma_{\mathrm{obs}}$ & $\sigma_{\text {sim }}$ \\
\hline Centro & 0,86 & 4,03 & 0,90 & 7,96 & 15,62 & 13,90 & 0,71 & $-4,60$ & 0,81 & 14,05 & 17,28 & 19,40 \\
\hline Copacabana & 0,84 & 1,72 & 0,90 & 7,87 & 14,46 & 12,40 & 0,66 & $-9,44$ & 0,72 & 14,92 & 15,74 & 19,64 \\
\hline São Cristóvão & 0,83 & $-6,67$ & 0,84 & 13,34 & 23,18 & 15,17 & 0,66 & $-7,60$ & 0,76 & 15,41 & 17,42 & 19,80 \\
\hline Tijuca & 0,89 & $-5,98$ & 0,89 & 8,61 & 18,27 & 14,16 & 0,65 & 2,08 & 0,79 & 15,64 & 18,14 & 19,58 \\
\hline Irajá & 0,88 & 1,44 & 0,93 & 8,58 & 18,52 & 15,92 & 0,68 & $-3,58$ & 0,80 & 15,31 & 17,96 & 20,01 \\
\hline Bangu & 0,89 & $-2,39$ & 0,91 & 10,26 & 21,27 & 15,18 & 0,51 & $-10,90$ & 0,75 & 19,08 & 18,33 & 20,11 \\
\hline Pedra de Guaratiba & 0,75 & 12,78 & 0,78 & 13,54 & 17,71 & 6,56 & 0,57 & 3,90 & 0,73 & 19,50 & 23,62 & 12,61 \\
\hline
\end{tabular}


valho, 2010; Pimentel et al., 2014). Vale ressaltar que as circulações de mesoescala são favorecidas quando o fluxo sinótico é fraco, o que acontece quando há atuação do ASAS na região. A Fig. 2 exemplifica a atuação da brisa marítima no litoral da região durante o período da tarde, quando é mais intensa. Utilizando os dados de vento registrados na estação de Pedra de Guaratiba, estação mais próxima do oceano, pode-se verificar o comportamento da mesma durante os 2 períodos de estudo. Durante o segundo período (Fig. 2 b), foram verificados ventos mais intensos vindos de sudeste e sudoeste, direção tipicamente associada a brisa marítima na RMRJ, como destacado por Dereczynski et al. (2009). Durante o primeiro período (Fig. 2 a), maiores intensidade de ventos oriundos da direção sudeste foram identificados. Porém, é valido destacar que devido à localização da estação meteorológica é possível que a mesma sofra influência da brisa marítima proveniente das duas direções (sudeste e sudoeste). Diferenças no posicionamento do ASAS e na temperatura da superfície do mar também podem influenciar no resultado final. Contudo, vale destacar que, principalmente, no segundo período, a intensidade dos ventos, mesmo em períodos quando a brisa marítima é mais intensa, é mais baixa, o que desfavorece dispersão dos poluentes. Isso tende a concentrar mais os poluentes em uma mesma região, o que reflete no aumento da concentração de poluentes.

A ocorrência de brisa marítima e terrestre foi identificada nos resultados do modelo WRF, através da grade de $4 \mathrm{Km}$, a partir da análise da direção e magnitude do vento e da razão de mistura. Os resultados do modelo indicam a ocorrência de brisa marítima no litoral, à tarde, durante ambos os períodos de estudo (exemplo na Fig. 3). Nota-se também que os horários (entre 12 e $18 \mathrm{HL}$ ) de atuação da

a)

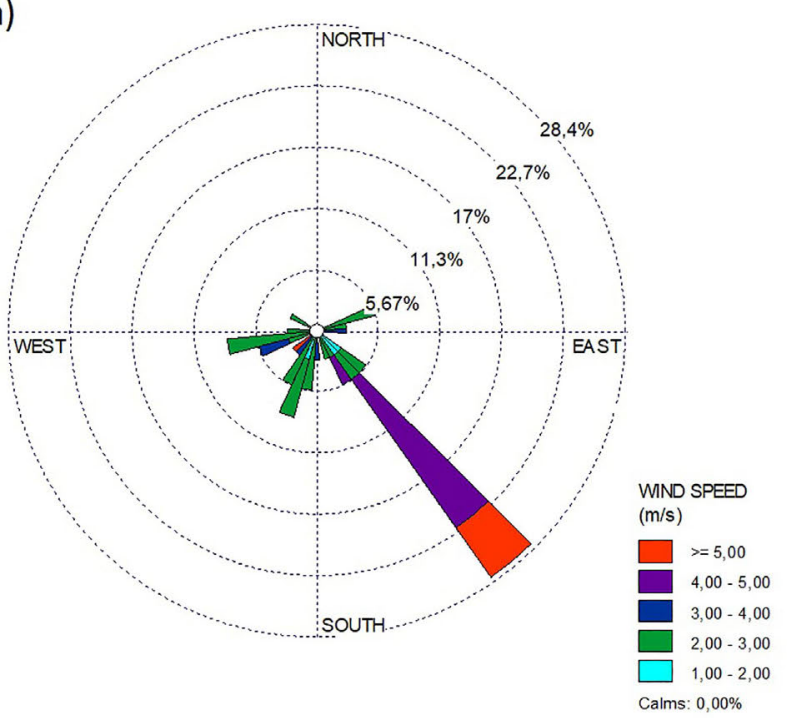

brisa marítima sobre a região coincidem com os maiores valores de razão de mistura. Na Fig. 3, correspondente ao dia 23 de setembro de 2015, é possível verificar uma configuração de brisa terrestre durante o horário das 09Z (06 $\mathrm{HL}$ ) em parte da região e o início da brisa marítima às $15 \mathrm{Z}$ (12 HL). Dereczynski et al. (2009) destacam que a brisa terrestre, na maior parte das vezes menos intensa do que a brisa marítima, em geral, é verificada durante a madrugada e início da manhã com inversão da direção dos ventos para o sentido continente-litoral, o que dificulta a dispersão de poluentes como $\mathrm{PM}_{10}$ e os precursores de $\mathrm{O}_{3}$ e os confina em regiões mais próximas ao litoral. Essa afirmação é corroborada pelos resultados encontrados a partir do modelo indicando que a atuação conjunta desses fatores colaboraram para a verificação de altas concentração de poluentes sobre a região.

Analisando os resultados da distribuição espacial da altura da camada limite planetária (CLP) e da temperatura do ar à $2 \mathrm{~m}$, é possível verificar que as horas dos dias com as maiores temperaturas, conforme o esperado, estão associadas a maiores valores da altura da CLP (exemplo na Fig. 4). Ressalta-se que o desenvolvimento da CLP interfere diretamente na mistura dos poluentes próximo a superfície terrestre, ou seja, quanto maior a altura da CLP maior a mistura próxima à superfície. Logo, a baixa altura da CLP identificada durante o período da madrugada e início da manhã podem ter um importante papel na manutenção de poluentes como o $\mathrm{PM}_{10}$ e os precursores do ozônio sobre a RMRJ durante os períodos de estudo, contribuindo para as altas concentrações de poluentes registradas. Na Fig. 4 (a), por exemplo, é possível observar valores mais baixos de altura da CLP, durante os dias $17 \mathrm{e}$ 22, na estação do Irajá; os dois dias correspondem aos

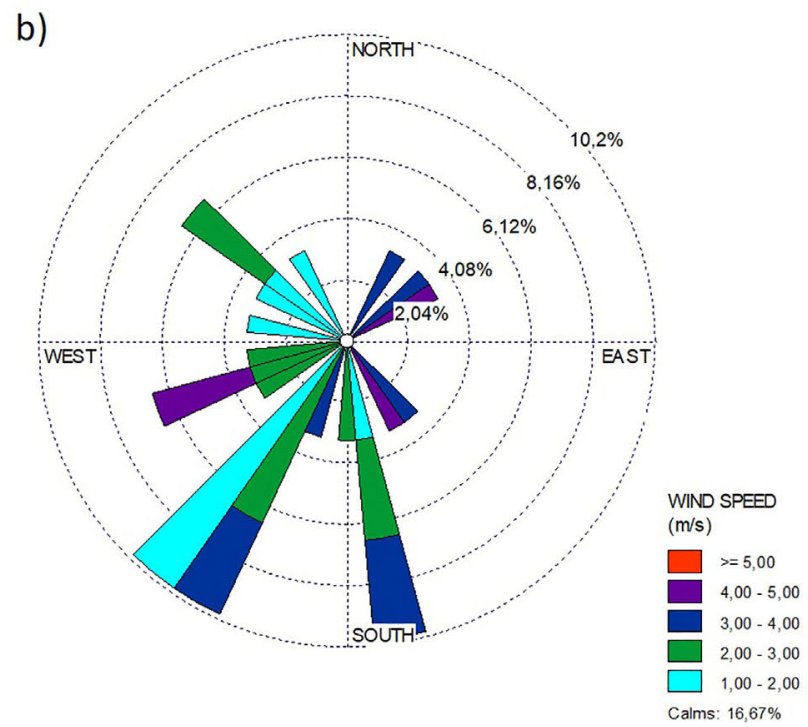

Figura 2 - Rosa dos Ventos entre as 13 e 18 horas, estação Pedra de Guaratiba do período entre (a) de 17/11/2013 a 22/11/2013 e (b) 23/09/2015 a 27/09/ 2015. 

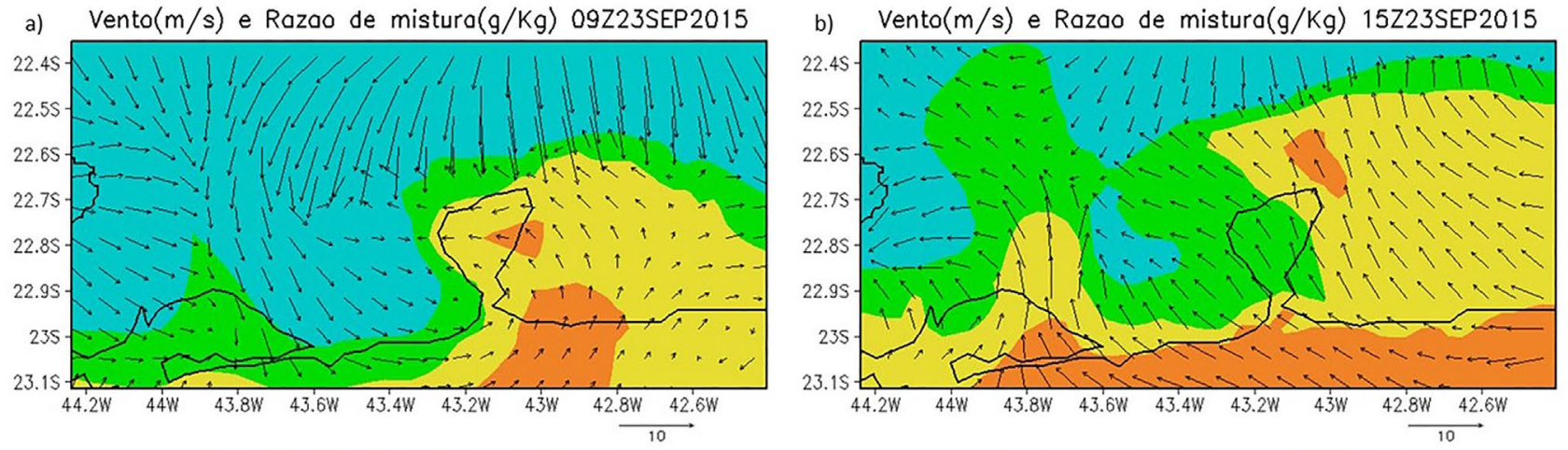

Figura 3 - Campo de Vento (m/s) e Razão de Mistura (g/kg) referente ao mês de setembro de 2015 (a) dia 23 a 09Z e (b) dia 23 a 15Z, simuladas através da grade de $4 \mathrm{~km}$, pelo WRF.

a)

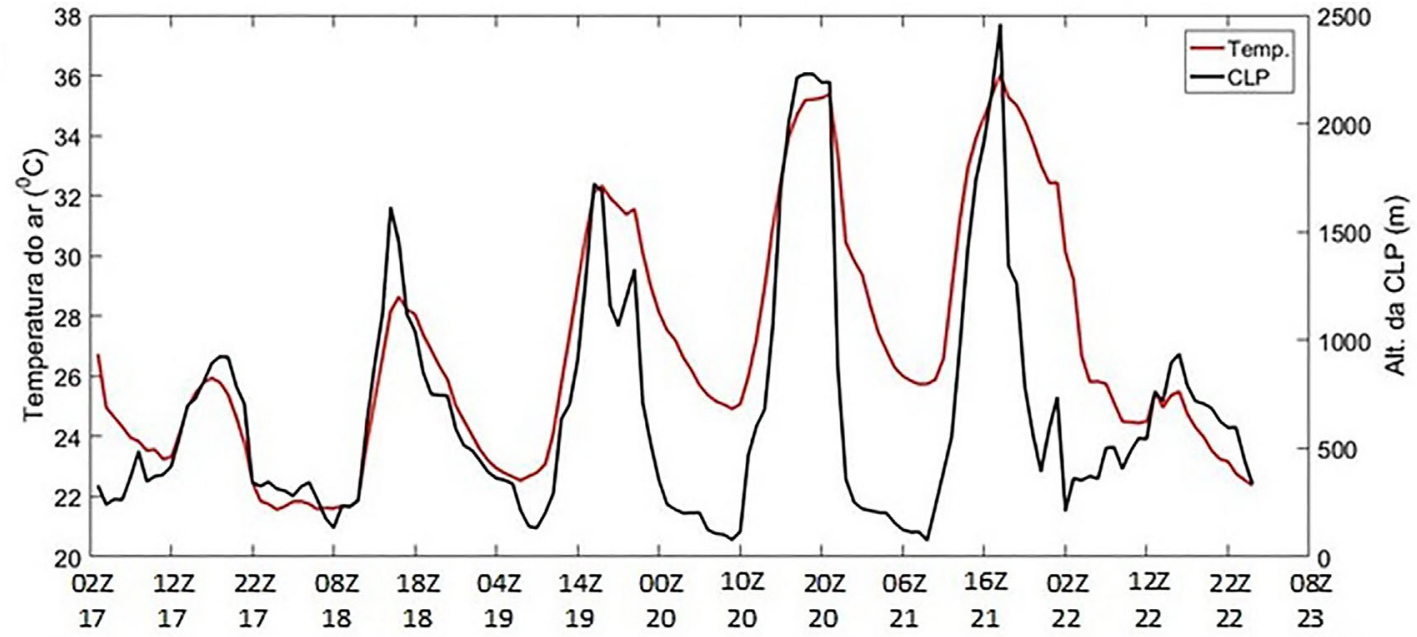

b)



Figura 4 - Altura da CLP e temperatura do ar ao longo dos períodos (a) de 17/11/2013 a 22/11/2013 e (b) 23/09/2015 a 27/09/2015, na estação do Irajá, simuladas através da grade de $4 \mathrm{~km}$, pelo WRF.

valores de maior concentrações de $\mathrm{PM}_{10}$ e de menor concentrações de $\mathrm{O}_{3}$, quando comparado com os demais dias do primeiro período de estudo. O mesmo é verificado na Fig. 4 (b), quando as menores de concentrações de $\mathrm{O}_{3}$ e as 
maiores concentrações de $\mathrm{PM}_{10}$, são identificadas durante os dias 23, 26 e 27, que coincidem com os menores valores de altura da CLP, quando comparado com os demais dias do segundo período. Vale destacar que os resultados obtidos encontram-se de acordo com o esperado, uma vez que baixos valores de altura da CLP são associados a maiores concentrações de poluentes primários. Isso, em geral, tende a manter as concentrações de ozônio mais baixas por conta de maiores concentrações de $\mathrm{NO}$, que atuam no consumo da molécula de $\mathrm{O}_{3}\left(\mathrm{O}_{3}+\mathrm{NO} \rightarrow \mathrm{O}_{2}+\mathrm{NO}_{2}\right)$.

\section{Conclusões}

Através desse estudo foi possível analisar e avaliar as condições meteorológicas em dois episódios de alta concentração de $\mathrm{PM}_{10}$ e $\mathrm{O}_{3}$ registrados na RMRJ que ultrapassaram os valores referências proposto pela OMS para uma boa qualidade do ar.

As simulações do modelo WRF de variáveis e condições meteorológicas ligadas aos episódios altas concentrações de $\mathrm{O}_{3}$ e $\mathrm{PM}_{10}$ na RMRJ revelaram boa concordância com os dados observados. Destacou-se em ambos os períodos analisados a atuação do ASAS, a ocorrência de baixa nebulosidade, principalmente, no primeiro período de estudo e altos valores de temperaturas. Foi possível observar pelos dados simulados a influência de brisa marítima durante a tarde e da brisa terrestre durante o início da manhã. Também foi possível verificar que as menores altura de CLP, estão associadas a alta concentrações de $\mathrm{PM}_{10}$ e baixas concentrações de $\mathrm{O}_{3}$. A aplicação dos índices estatísticos revelou que o modelo representou com coerência as variáveis temperatura do ar e umidade relativa do ar.

\section{Agradecimentos}

Agradecemos ao CNPq (Bolsa de mestrado Processo $\mathrm{n}^{\circ}$ 133772/2017) e a CAPES (recursos através do PROAP) pelo apoio financeiro necessário para a realização deste projeto.

\section{Referências}

CARVALHO, V.S.B. O Impacto das Megacidades Sobre a Qualidade do Ar: Os Casos das Regiões Metropolitanas de São Paulo e do Rio de Janeiro. Tese de Doutorado em Meteorologia, Instituto de Astronomia, Geofísica e Ciências Atmosféricas, Universidade de São Paulo, São Paulo, 2010.

CARVALHO, V.S.B.; FREITAS, E.D.; MAZZOLI, C.R.R.; ANDRADE, M.F. Avaliação da influência de condições meteorológicas na ocorrência e manutenção de um episódio prolongado com altas concentrações de ozônio sobre a Região Metropolitana de São Paulo, Brasil. Revista Brasileira de Meteorologia, v. 27, n. 4, p. 458-469, 2012.
DERECZYNSKI, C.P.; OLIVEIRA, J.S.; MACHADO, C.O. Climatologia da precipitação no município do Rio de Janeiro. Revista Brasileira de Meteorologia, v. 24, n. 1, p. 24-38, 2009.

ELBIR, T. Comparison of model predictions with the data of an urban air quality monitoring network in Izmir, Turkey. Atmospheric Environment, v. 37, p. 2149-2157, 2003.

FAJERSZTAJN, L.; SALDIVA, P.; PEREIRA, L.A.A.; LEITE, V.F.; BUEHLER, A.M. Short-term effects of fine particulate matter pollution on daily health events in Latin America: a systematic review and meta-analysis. International Journal of Public Health, v. 2, n. 1, p. 1-10, 2017.

GALLARDO, L.; ANDRADE, M.F.; CARVALHO, V.S.B. Chapter 4: South America. In: Tong Zhu, Megan Melamed, David Parrish, Michael Gauss, Laura Gallardo Klenner, Mark Lawrence, Abdourahamane Konare, Cathy Liousse. In: Impacts of Megacities on Air Pollution and Climate. World Meteorological Organization, Geneva, 309 p., 2012.

INSTITUTO ESTADUAL DO AMBIENTE (INEA). Relatório Anual de Qualidade do Ar do Estado do Rio de Janeiro. INEA, Rio de Janeiro, 2015.

INSTITUTO ESTADUAL DO AMBIENTE (INEA). Relatório Anual de Qualidade do Ar do Estado do Rio de Janeiro. INEA, Rio de Janeiro, 2014.

LAWRENCE, M.G.; BUTLER, T.M.; STEINKAMP, J; GURJAR, B.R.; LELIEVELD, J. Regional pollution potentials of megacities and other major population centers. Atmospheric Chemistry and Physics. v. 6, p. 13323-13366, 2006.

ORGANIZAÇÃO MUNDIAL DE SAÚDE (OMS). Air Quality Guidelines for Particulate Matter, Ozone, Nitrogen Dioxide and Sulfur Dioxide: Global Update 2005. WHO/ SDE/PHE/OEH/06.02, 2006.

PIELKE, R.A. Mesoscale meteorological modeling. Second Edition. International Geophysics Series, v. 78, n. 1, p. 676, 2002.

PIMENTEL, L.C.G., CORRÊA, E.B., MARTON, E.; CATALDI, M.; NOGUEIRA, E. Influência dos parâmetros de configuração do modelo CALMET sobre a simulação da circulação atmosférica na região metropolitana do Rio de Janeiro. Revista Brasileira de Meteorologia, v. 29, n. 4, p. 579-596, 2014.

SANTOS, T.C.; CARVALHO, V.S.B.; REBOITA, M.S. Avaliação da influência das condições meteorológicas em dias com altas concentrações de material particulado na Região Metropolitana do Rio de Janeiro. Engenharia Sanitária e Ambiental, v. 21, p. 307-313, 2016.

SILVEIRA, W.W. Impacto da Estabilidade Atmosférica na Concentração de Poluentes na Região Metropolitana do Rio de Janeiro. Dissertação de Mestrado em Meio Ambiente e Recursos Hídricos, Universidade Federal de Itajubá, Itajubá, 87 p., 2019.

SKAMAROCK, W.C.; KLEMP; J.B.; DUDHIA, J.; GILL, D.O.; BARKER, D.M.; DUDHA, M.G.; HUANG, X.; WANG, W.; POWERS, Y. A description of the advanced research WRF Ver.30. NCAR Technical Note. NCAR/TN- 475 +STR. National Centre for Atmospheric Research, Boulder, CO, p. 133, 2008. 
TAKANO, A P.C.; JUSTO, L.T.; DOS SANTOS, N.V.; MARQUEZINI, M.V.; DE ANDRÉ, P.A.; DA ROCHA, F.M.M.; PASQUALUCCI, C.A.; BARROZO, L.V.; SINGER, J.M.; DE ANDRÉ, C.D.; SALDIVA, P.H.N.; VERAS, M.M.

Pleural anthracosis as an indicator of lifetime exposure to urban air pollution: An autopsy-based study in Sao Paulo. Environmental Research, v. 173, p. 23-32, 2019.

WILKS, D.S. Statistical Methods in the Atmospheric Sciences, Academic Press, San Diego, p. 467, 1995.

\section{Endereços de Internet}

Diretoria de Hidrografia e Navegação da Marinha do Brasil, https://www.marinha.mil.br/dhn.

National Climatic Data Center do National Oceanic and Atmospheric Administration, https://www.ncdc.noaa.gov/ nomads.

Rede de Meteorologia do Comando da Aeronáutica, https:// www.redemet.aer.mil.br/.

License information: This is an open-access article distributed under the terms of the Creative Commons Attribution License (type CC-BY), which permits unrestricted use, distribution and reproduction in any medium, provided the original article is properly cited. 